\title{
Introduction to the Special Focus Column
}

\author{
Subin Xu \\ Chair of the EAAC 2017 Organising Committee \\ School of Architecture, Tianjin University, Tianjin, China \\ Email:1421750993@qq.com
}

The study of East Asian architecture is pushing the frontiers of scholarly research at a global level, attracting the attention of a growing number of scholars and providing insights to a greater number of research topics. The recent history of the International Conference on East Asian Architectural Culture (hereinafter referred to as EAAC) is a witness of such development. Originally proposed in 2000; the EAAC was first officially launched in 2002 and organised since then once every two years. The participants of the first editions of the Conference mainly consisted of researchers in the field of architecture history and theory from China, Japan and South Korea. With the gradual increasing influence of EAAC, more architecture scholars from other regions in Asia such as Taiwan, Hong Kong and Southeast Asia were also involved. Later on, the conference further stepped in Singapore and Hong Kong, which enhanced the research and communication about architecture history and heritage conservation in East and Southeast Asia.

From 13 October through 17 October 2017, Tianjin University in China held the last edition of EAAC. Under the theme of 'East Asia Architecture: Value, Inheritance \& Dissemination', it was a major event attended by international scholars that included relevant speeches, parallel sessions and a round-table discussion. Six renowned scholars as Chen Tongbin (China Architecture Design \& Research Group, Beijing), Fu Chao-Ching (Cheng Kung University, Tainan), Lee Sang-hae (Kookmin University, Seoul), Shimizu Shigeatsu (Kyoto Institute of Technology, Kyoto), Tay Kheng Soon (National University of Singapore, Singapore) and Charlie Qiuli Xue (City University of Hong Kong, Hong Kong) were invited to address the keynote speeches, whereas the round-table meeting was organised around the topic of 'Contestation and Cooperation: Architectural History in Education and Practice', which clearly points towards the future of the discipline in relationship with its intrinsic development, as well as with society itself.

An insight into the numbers of EAAC 2017 can provide useful information about the current tendencies in research

and practice in the region. The organising committee of the Conference received 490 abstracts among which 292 ones were selected. Meanwhile, the actual submitted papers counted 133, and their authors were all arranged to parallel sessions categorised into the four subthemes: (1) Historical and Theoretical Research; (2) Conservation Methodology and Technology; (3) Adaptive Reuse; and (4) Community Design. The number of papers for each subtheme was 88 , 20, 13 and 9 respectively; a distribution that allows us to make an immediate analysis: contributions for the topic of 'Historical and Theoretical Research' exceeded the others; while on the other hand, the papers devoted to the topics of 'Conservation Methodology and Technology' were relatively fewer than expected. This offers an important contrast with the growing interest in heritage conservation in the East Asia region and China especially after the year 2000, for which the Built Heritage Journal is also an important witness. Besides, the number of papers received for the newly-added topic of 'Community Design' is also less than initially expected, which means that the scholarly approach to this aspect requires further attention in the future.

The geographical distribution of the papers also spoke of the rising importance of these studies in China. Scholars from China took the absolute advantage with 95 ones including papers by Hong Kong and Taiwan scholars with 18. Adding to them, there were 16 papers submitted by authors from Japan, as well as 15 papers from other countries. Even though a deeper analysis may originate from these numbers and from other aspects of the Conference, this quick panorama is useful to depict the challenges for the celebration of the next EAAC Conference, which will be held in Tongji University, Shanghai, in 2019, reinforcing the commitment that this institution is showing with the field of built heritage conservation.

A prelude to the major scientific meeting that will be taking place in Tongji in the next year is the issue of Built Heritage that the readers have now in their hands. Thanks to the great support of Tongji University, three of the most 
relevant papers presented at the EAAC in the field of built heritage conservation have been selected for publication in this Special Focus column of Built Heritage. They present the up-to-date research about built heritage study and conservation from three different perspectives that include the recent topic of modern industrial heritage; together with specific visions into official monumental architecture, and vernacular architecture.

The first paper contributed by Subin $\mathrm{Xu}$ and Nobuo Aoki briefly summarises the results from 'Comprehensive Research on the Preservation of the Modern Industrial Heritage in China, a major program of the National Social Science Funds of China. Conducted for five years, this study is a relatively systematic and interdisciplinary exploration on Chinese industrial heritage conservation that integrates multiple dimensions including the history of industrial technology and communication, information acquisition and heritage management, the evaluation, protection and re-utilisation of industrial heritages, cultural industry; among others.

The second paper contributed by Hong Yang, Jiawei Xie and Lifang Ji analyses the decorative polychrome painting of the architectural members (caihua) that has survived in the Hall of Mental Cultivation (Yangxindian) in the Forbidden City, Beijing. Combining information gained through on-site surveys and scaled drawings executed during a recent conservation project with evidence from historical records and other sources, this study discovers the remaining of decorative polychrome painting that can be dated to four different historical phases from the late Ming to late Qing dynasties.

The third paper contributed by Congcong Ren, Ruchen Bian and Simiao Li examines the whole construction process of the traditional chuandou dwellings, which is prevailing in the mountainous countryside of the Dong minority area in the Southwest of China. By following the work of a group of chief carpenters, it also provides a detailed observation of the relationship between ritual and technology: How the two can be combined, how they influence the appearance of houses and how one can master them and pass down their knowledge and know-how to future generations of carpenters.

Reflecting on this variety, this Special Focus column of Built Heritage aims to help international scholars obtain a better understanding of the recent development and achievements of built heritage study and conservation in China. 